\title{
Quest for superheavy nuclei
}

\author{
P.H. Heenen ${ }^{1}$ and W. Nazarewicz ${ }^{2-4}$ \\ ${ }^{1}$ Service de Physique Nucléaire Théorique, U.L.B.-C.P.229, B-1050 Brussels, Belgium \\ ${ }^{2}$ Department of Physics, University of Tennessee, Knoxville, Tennessee 37996 \\ ${ }^{3}$ Physics Division, Oak Ridge National Laboratory, Oak Ridge, Tennessee 37831 \\ "Institute of Theoretical Physics, University of Warsaw, ul. Hol.za 69, PL-00-681 Warsaw, Poland
}

$T$ he discovery of new superheavy nuclei has brought much $I$ excitement to the atomic and nuclear physics communities. Hopes of finding regions of long-lived superheavy nuclei, predicted in the early 1960s, have reemerged. Why is this search so important and what new knowledge can it bring?

Not every combination of neutrons and protons makes a stable nucleus. Our Earth is home to 81 stable elements, including slightly fewer than 300 stable nuclei. Other nuclei found in nature, although bound to the emission of protons and neutrons, are radioactive. That is, they eventually capture or emit electrons and positrons, alpha particles, or undergo spontaneous fission. Each unstable isotope is characterized by its half-life $\left(T_{1 / 2}\right)$ - the time it takes for half of the sample to decay. Isotope half-lives range from less than a thousandth of a second to billions of years. Many radioactive nuclei may have half-lives comparable to or longer than the age of the Earth ( 4.5 billion years). Examples are the actinide nuclei ${ }^{232} \mathrm{Th}\left(T_{1 / 2}=1 \cdot 10^{10}\right.$ years $),{ }^{235} \mathrm{U}\left(T_{1 / 2}=7 \cdot 10^{8}\right.$ years), or ${ }^{239} \mathrm{U}\left(T_{1 / 2}=4 \cdot 10^{9}\right.$ years). Short-lived isotopes cannot be found naturally on Earth because they have long decayed since our planet was formed. Yet, thousands of short-lived isotopes are continually created in the cosmos. Their existence may be fleeting, but they play a crucial role in the ongoing formation of the elements in the Universe. The properties of most rare isotopes are unknown and can be only inferred, with considerable uncertainty, from theoretical calculations.

Figure 1 shows bound nuclear systems as a function of their proton number $Z$ (vertical axis) and their neutron number $N$ (horizontal axis). The black squares represent the stable or very long-lived nuclei. They form the so-called "valley of stability". The yellow region indicates shorter-lived nuclei that have been produced and studied in laboratories. By adding either protons or neutrons, one moves away from the valley of stability, finally reaching the drip lines where nuclear binding ends because the forces between neutrons and protons are no longer strong enough to hold these particles together.

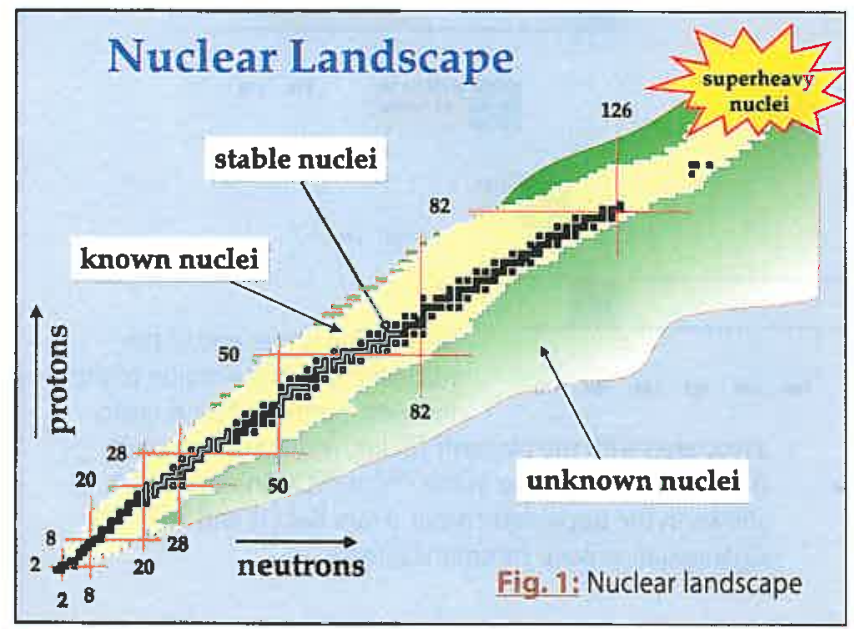

The superheavy elements mark the limit of nuclear mass and charge; they inhabit the upper right corner of the nuclear landscape, but the borderlines of their territory are unknown. The stability of the superheavy elements has been a longstanding fundamental question in nuclear science. How can they survive the huge electrostatic repulsion? What are their properties? How large is the region of superheavy elements? We do not know yet all the answers to these questions. This short article presents the current status of research in this field.

\section{Historical Background}

The quest for superheavy nuclei began in the 1940s with the synthesis of atomic nuclei with a number of protons greater than uranium ( $Z=92)$. In 1940 , neptunium $(Z=93)$ and plutonium $(Z=94)$ were discovered. This was followed by the synthesis of americium $(Z=95)$ and curium $(Z=96)$ in 1944 , berkelium $(Z=97)$ in 1949 , californium $(Z=98)$, einsteinium $(Z=99)$ and fermium $(Z=100)$ in 1952, and mendelevium $(Z=101)$ in 1955. All these elements were produced by intense neutron irradiations or by proton, deuteron, or helium (alpha particle) bombardment in a cyclotron. Some of these isotopes have been produced in sizable amounts. Einsteinium and fermium were discovered in the debris from the thermonuclear explosion conducted at Eniwetok Atoll in the Pacific Ocean. Amazingly, the fermium nucleus was made through the capture of 17 neutrons by ${ }^{238} \mathrm{U}$ followed by the subsequent beta decays. In nature, a similar process (the so-called r-process, believed to be taking place in supernova) is responsible for the synthesis of heavy elements.

Still heavier elements (transuraniums) were produced in heavy-ion accelerators by fusing heavy actinide targets (plutonium-californium) with light ions of carbon (nobelium, $Z=102$, 1958; ruthefordium, $Z=104,1969$ ), boron (lawrencium, $Z=103$, 1961 ), neon (dubnium, $Z=105,1967$ ), and oxygen (seaborgium, $Z=106,1974$ ). In order to go heavier, to compensate the decrease of the proton-to-neutron ratio with mass, fusion of nuclei with the largest possible surplus of neutrons had to be used. In the 70s, "cold fusion" reactions involving medium-mass projectiles with $Z \geq 24$ and lead or bismuth targets were introduced and replaced "hot fusion" reaction with actinide targets. This enabled the discovery of bohrium $(Z=107,1976)$, hassium $(Z=108,1984)$, meitnerium $(Z=109,1982)$, ununnilium $(Z=110,1994)$, unununium $(Z=111,1994)$, and ununbium $(Z=112,1996)$. The last three elements are still unnamed. So far, they carry temporary names derived directly from the atomic number according to the systematic nomenclature of the International Union of Pure and Applied Chemistry (IUPAC).

Most of the heaviest elements were found in three "heavy element factories": Lawrence Berkeley Laboratory in Berkeley (USA), Joint Institute for Nuclear Research in Dubna (Russia), and Gesellschaft für Schwerionenforschung (GSI) near Darmstadt (Germany). After much discussion about the priority for their synthesis, IUPAC 1997 has accepted names up to $Z=109$. At 
that time, nuclei with $Z=110,111$, and 112 , discovered at GSI, were already known. The lifetimes of the heaviest elements were found to be very short. For instance, element $Z=112$, investigated using the reaction ${ }^{70} \mathrm{Zn}+{ }^{208} \mathrm{~Pb} \rightarrow{ }^{277} \mathrm{Uub}+1 \mathrm{n}$, turned out to have a half life of only about $280 \mu \mathrm{sec}$. The corresponding fusion cross section is extremely small, $1 \mathrm{pb}$. Since the production cross section was found to be rapidly decreasing with the atomic number, see Fig. 2, it was then concluded that it would be very difficult to reach still heavier elements.

As far as theory is concerned, major advances were made in 1966 , when the first realistic theoretical calculations for the superheavy nuclei were carried out. These early calculations were based on the shell

correction (or: macroscopic-microscopic) method in which the total binding energy was decomposed into a smooth part (approximated by a macroscopic liquid drop model) and a microscopic quantal shell correction term strongly oscillating with the number of nucleons and reflecting the energy stabilization due to the presence of nucleonic shells. Such calculations predicted the nucleus with $Z=114, N=184$ ( ${ }_{114}^{298} \mathrm{Uuq}$ ) to be a doubly magic center of an island of long-lived superheavy nuclei. This result stayed practically unchallenged until the late 1990 s where more refined models, based on self-consistent mean-field theory and realistic effective nucleon-nucleon interactions, started to be systematically applied to superheavy nuclei. In another frontier of superheavy element research, in quantum chemistry, relativistic effects in molecules containing heavy elements have been studied intensively since the mid 1970s, when it became apparent that they have to be included in the description of compounds containing heavy elements.

\section{New discoveries}

The last three years (1999-2001) have brought a number of experimental surprises which have truly rejuvenated the field [1]. In January 1999, scientists from Dubna reported the synthesis of one atom of element 114 ( ${ }_{114}^{298} \mathrm{Uuq}$ ) in a "hot fusion" reaction between a ${ }^{48} \mathrm{Ca}$ beam and a ${ }^{244} \mathrm{Pu}$ target. This discovery was followed by three other reports, also from Dubna. First, using the ${ }^{242} \mathrm{Pu}\left({ }^{48} \mathrm{Ca}, 3 \mathrm{n}\right)$ reaction, they produced ${ }^{287} \mathrm{Uuq}$. In 1999 , the synthesis of yet another isotope of $Z=114$, the even-even ${ }^{288} \mathrm{Uuq}$, was reported. The two $\alpha$ decay sequences were confirmed afterwards (July 2000, May 2001) by the observation of three $\alpha$ chains associated with the element $Z=116\left({ }_{116}^{292} \mathrm{Uuh}\right)$ in the ${ }^{248} \mathrm{Cm}\left({ }^{48} \mathrm{Ca}, 4 \mathrm{n}\right)$ reaction. According to the Dubna analysis, the resulting a-decay chain goes through ${ }^{288} \mathrm{Uuq},{ }^{284} \mathrm{Uub}$, and ${ }^{280} \mathrm{Uun}$, which subsequently fissions. In neither of these cases do the a chains end up at a known isotope so a firm assignment could not be made. Considering the exponential trend seen in Fig. 2 for $Z \leq 114$, it actually

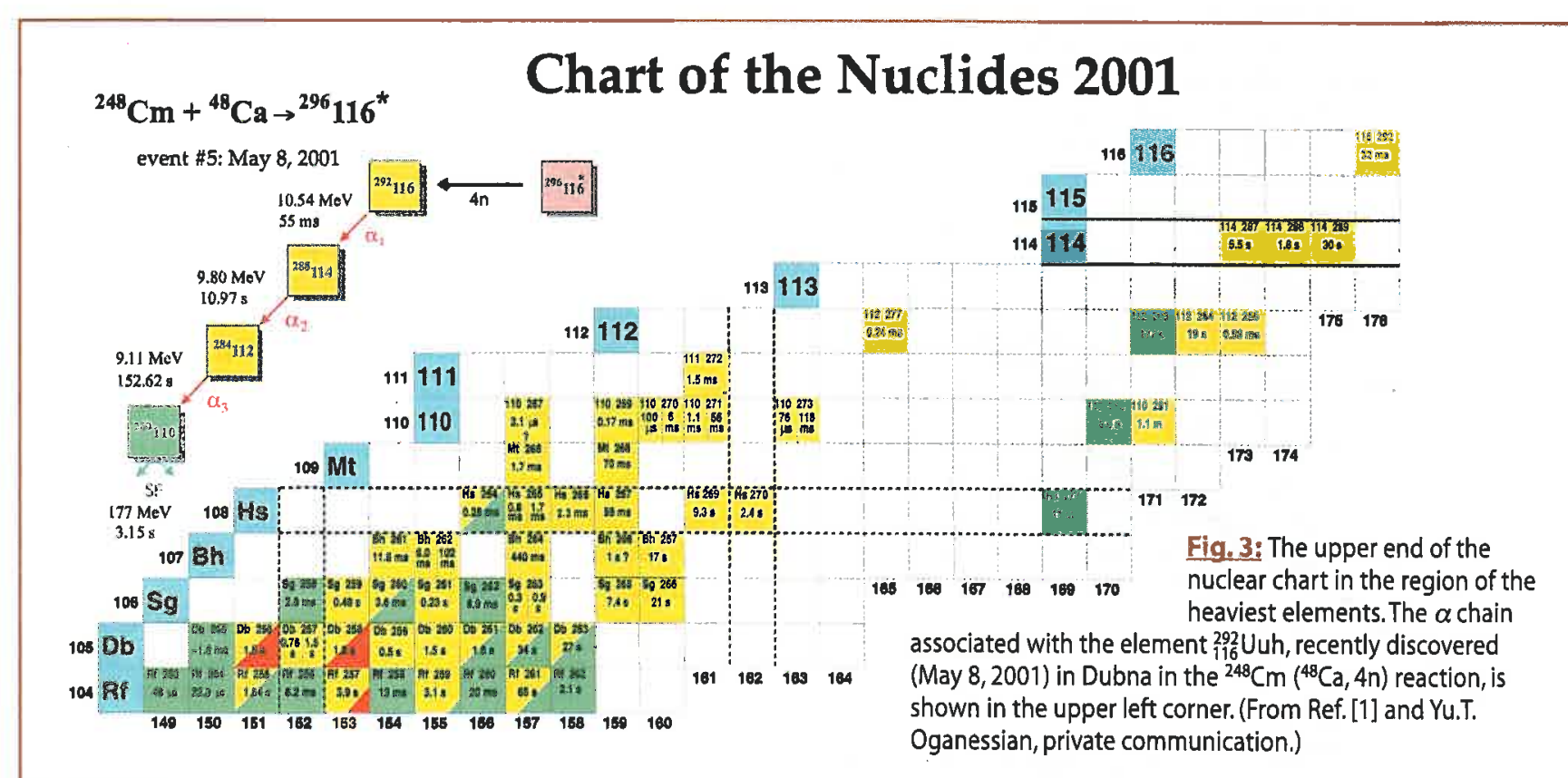


came as a great surprise that hot fusion reactions with a ${ }^{48} \mathrm{Ca}$ beam could produce a significant number of events. Another surprising (and exciting) result of the Dubna experiments was that nuclei which were produced have fairly long lifetimes. For instance, the lifetimes reported for the elements ${ }^{284} \mathrm{Uub}$ and ${ }^{280}$ Uun are many orders of magnitude longer than those of the isotopes with $Z \leq 112$ previously discovered at GSI.

Progress has also been made in the region previously explored by GSI. The GSI group found a new even-even isotope of ${ }^{270} \mathrm{Uun}$, and also ${ }^{272} \mathrm{Uuu}$ and ${ }^{277} \mathrm{Uub}$. A group led by scientists from the Paul Scherrer Institute (PSI) observed the ${ }^{269,270} \mathrm{Hs}$ decay chains at GSI, thus convincingly confirming the data on ${ }^{277} \mathrm{Uub}$ for which ${ }^{269} \mathrm{Hs}$ is a member of the decay chain. New isotopes of ${ }^{266,267} \mathrm{Bh}$ have been found at Berkeley. As far as chemistry is concerned, a tremendous achievement and a true tour-de-force were the first chemical studies of seaborgium, bohrium, and hassium (see below). Figure 3 shows the upper end of the nuclear chart as of 2001 .

Physics at the limit of cross sections is difficult and challenging. The synthesis of the new element $Z=118$ ( ${ }^{293} \mathrm{Uuo}$ ), announced in 1999 by the Berkeley group, has been retracted two years later. The history of heavy element research remembers other cases of elements discovered and gone, and sometimes discovered again. A team working in Stockholm at the Nobel Institute of Physics reported in 1957 an isotope with atomic number 102. The group proposed the name Nobelium in honor to Alfred Nobel. In 1958 a group at Berkeley reported that they were unable to reproduce this work, findings agreed by a Russian group at Dubna. But the name stuck...

\section{Structure of the superheavy nuclei}

The structure of superheavy elements results from the interplay between the strong and electromagnetic forces. Unlike in lighter nuclei, the Coulomb interaction cannot be treated as a small perturbation atop the dominating nuclear interaction; it does influence significantly proton and neutron distributions[2].

The stability of heavy and superheavy elements has been a longstanding fundamental question in nuclear science. Theoretically, it has been shown that the mere existence of nuclei with $Z>102$ is entirely due to quantal shell effects. Indeed, in the classical liquid drop picture, the shape of a nucleus is governed by an interplay between surface tension (proportional to $\mathrm{A}^{2 / 3}$, trying to make the nucleus spherical) and Coulomb repulsion (proportional to $Z^{2} / A^{1 / 3}$, trying to make the nucleus deformed). At large values of $Z^{2} / A$, the Coulomb force is so strong that the nuclear liquid drop becomes unstable to surface distortions and it fissions spontaneously. It is the stabilization brought by quantal shell effects that is responsible for the superheavies.

In spite of an impressive agreement with experimental data for the heaviest elements, theoretical uncertainties are large when extrapolating to unknown regions of the nuclear chart. In particular, there is no consensus among theorists with regard to the center of the shell stability in the superheavy region. Since in these nuclei the single-particle level density is relatively large, small shifts in the position of single-particle levels (e.g., due to the Coulomb or spin-orbit interaction) can be crucial for determining the shell stability of a nucleus. While most macroscopic-microscopic (non-self-consistent) approaches pre$\operatorname{dict} Z=114$ to be magic, most self-consistent calculations suggest that the center of the proton shell stability should be moved up to higher proton numbers, $Z=120,124$, or 126 . For the neutrons, most non-relativistic calculations predict magic gaps at $N=184$ while the relativistic mean-field theory yields $N=172$ - due to a slightly different spin-orbit interaction. The experimental determination of superheavy nuclei from this region will thus be of extreme importance for pinning down the fundamental question of the spin-orbit force.

Does it actually make sense to talk about "magic superheavy nuclei"? A recent theoretical work [3] sheds a new light on this question. According to calculations, the patterns of single-particle levels are significantly modified in the superheavy elements. Firstly, the overall level density grows with mass number $A$, as $\propto$ $A^{1 / 3}$. Secondly, no pronounced and uniquely preferred energy gaps appear in the spectrum. This shows that shell closures which are to be associated with large gaps in the spectrum are not robust in superheavy nuclei. Indeed, the theory predicts that beyond $Z=82$ and $N=126$ the usual localization of shell effects at magic numbers is basically gone. Instead the theory predicts fairly wide areas of large shell stabilization without magic gaps. This is good news for experimentalists: there is a good chance to reach shell-stabilized superheavy nuclei using a range of beamtarget combinations.

Some of the observed $\alpha$-decay chains attributed to elements 112 and 114 are believed to link odd- $N$ nuclei. The presence of an odd neutron adds to a theoretical uncertainty. Since the singleparticle level density of transactinides is large, there are many candidates for low-lying one-quasiparticle states in parent and daughter nuclei. Moreover, many alpha transitions are structurally forbidden since the properties of one-quasiparticle excitations in parent and daughter nuclei often differ dramatically. As an example, Fig. 4 shows the predicted a-chains for the nucleus ${ }^{277} 112$ found at GSI. The Hartree-Fock-Bogoliubov calculations explain two observed a branches, attributed experimentally to ${ }^{273} 110$, in terms of transitions between negative parity and positive parity single-neutron orbitals.

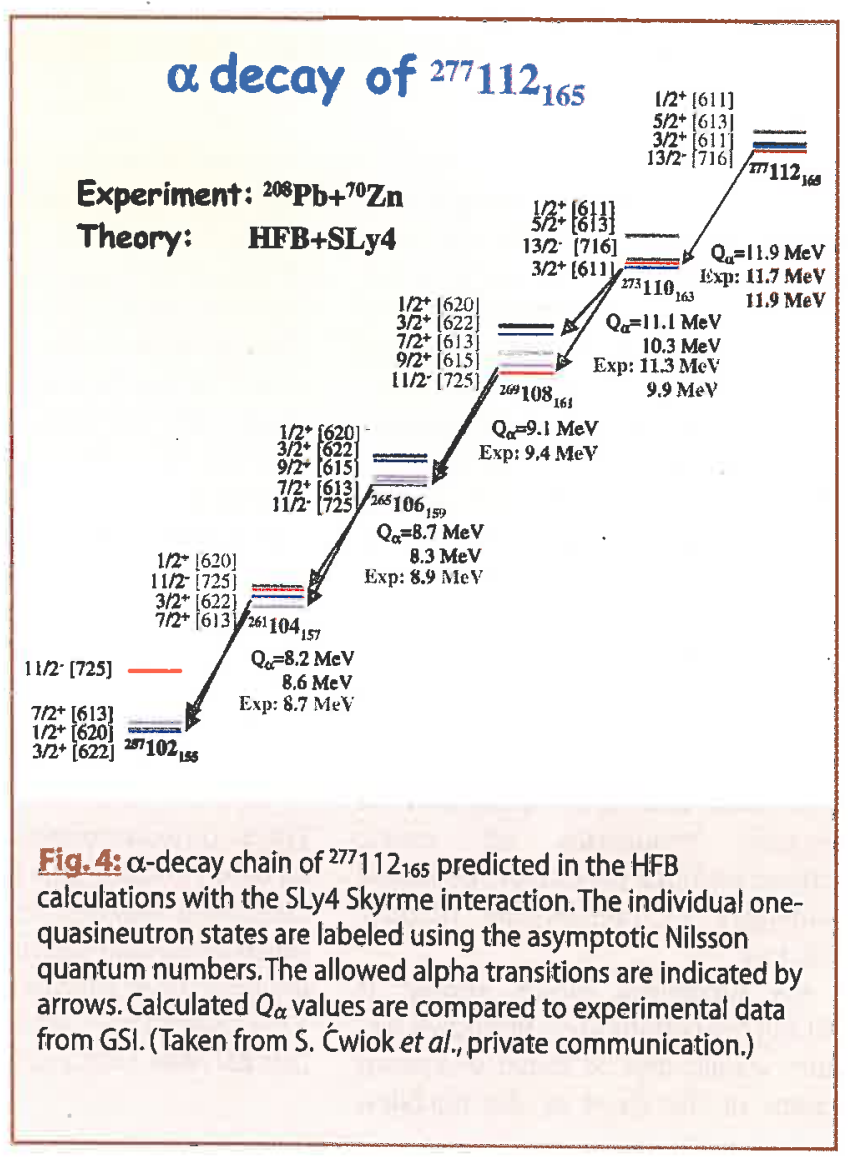




\section{Chemical properties of superheavy elements}

Since atomic relativistic effects scale approximately with $Z^{2}$, chemical properties of transactinides cannot be properly described by non-relativistic quantum mechanics. However, experimental tests of relativistic effects and of anticipated deviations from the periodic table of the elements in the superheavy region are extremely difficult because the single atom chemistry requires half-lives of the order of one second, i.e., much longer than those of the heaviest elements found so far. Another obstacle is very small production rates: a few events per day for hassium and less for heavier elements. In spite of these difficulties, there has been major progress in the chemical characterization of transactinides. Special techniques, such as gas phase separation, have been devised to perform one atom-at-a-time chemistry and to deduce thermodynamical properties of new elements [4].

In 1993, chemical studies of dubnium demonstrated that it does not behave like tantalum, the element from the group to which dubnium was supposed to belong. On the contrary, the following chemical studies of Sg (year 1997), Bh (2000), and Hs (2001) confirmed relativistic calculations predicting their behavior to coincide with that expected on the basis of their positions in the periodic table. Hassium, for instance, forms a gaseous oxide similar to that of osmium, which places it in group 8. As of today, the periodic table has been experimentally established up to element 108. However, preparations are under way to carry out gas chemistry of element 112, which instead of behaving like its lighter transition metal homologs $(\mathrm{Zn}, \mathrm{Cd}, \mathrm{Hg})$ is expected to have properties of a noble gas like $\mathrm{Rn}$ or $\mathrm{Xe}$; a first attempt to chemically identify element $Z=112$ has recently been carried out in Dubna. Still heavier elements, predicted to be noble volatile metals, are also excellent candidates for gas chemical studies, provided that their half-lives are sufficiently long. In particular, there is hope of employing chemical methods for the firm identification of the alpha-chains of long-lived isotopes of $Z=114$ and 116 observed in Dubna.
First predictions of the chemistry of superheavy elements were made in 1971, and the first relativistic molecular calculations for systems involving transactinide elements were published in 1977. Since then, calculations based on the relativistic quantum theory have been considerably improved and applied to many atoms and molecules [5]. Relativistic effects in transactinides are crucial and show up in several ways. Most importantly, $s$ and $p_{1 / 2}$ atomic orbitals contract relativistically. The shrinking of the inner shells results in an increased screening of the nuclear charge, and this gives rise to an expansion of the $p_{3 / 2}$ and of higher angular momentum orbitals. Another relativistic effect is a change in the spin-orbit coupling. Both can produce drastic rearrangements of orbital levels. That is what is predicted to happen for element 112. Recent calculations indicate that the $7 s$ orbital should be shifted below the $6 d_{5 / 2}$ orbital due to relativistic effects. It is the large relativistic stabilization of its valence $7 s$ orbital, combined with its closed shell electron configuration, that has led to the prediction that element 112 is chemically inert.

Chemistry of the superheavy elements with $Z>118$ is believed to show relativistic effects that are so large that comparison with lighter elements or nonrelativistic results is meaningless.

\section{Borders of the superheavy region}

Where are the borders of the superheavy region? What are the properties of the heaviest nuclei that can be bound (at least, in theory), in spite of the huge disruptive Coulomb force?

As a consequence of their saturation properties, nuclear forces favor values of the internal density close to the saturation density of nuclear matter. On the contrary, since the Coulomb interaction tends to increase the average distance between protons, the Coulomb energy is significantly lowered by either the creation of a central depression or by deformation, or by both. Based on this general argument, one expects the formation of voids in heavy nuclei. Recently, the subject of exotic (bubble, toroidal, band-like) configurations in nuclei with very large atomic numbers has been revisited by self-consistent calculations.
Box 1: The nucleus is a complicated many-body system. The strong forces describing heavy nuclei are strongly affected by the in-medium effects; they are usually approximated by phenomenological effective densitydependent interactions fitted to a few key nuclear properties. This strategy, well known in condensed matter physics, turned out to be extremely successful. The self-consistent density functional theories (relativistic and non-relativistic) describe bulk nuclear properties such as masses, radii, shapes, as well as nuclear excitations. A number of effective interactions have been introduced and their capability to describe properties of exotic radioactive nuclei is one of the major challenges in present-day nuclear structure.

Any theoretical model aiming at making predictions in an unknown territory should first be tested in known regions of the chart of the nuclides.
Since the main decay mode for the known heaviest elements is $\alpha$ decay, one of the crucial quantities determining lifetimes of the heaviest and superheavy elements is the $\alpha$-decay energy. The $Q_{\alpha}$ values for the heaviest elements calculated with the self-consistent model with the SLy4 effective interaction are displayed in Fig. 5. They are compared with known $\alpha$-decay energies. It is seen that the agreement with experimental data (including the recent data shown in Fig. 3 ) is very good, and this suggests that calculations such as those shown on Fig. 5 can be used to predict properties of unknown superheavy nuclei.

Fig. 5: $Q_{a}$ values for even-even nuclei with $96 \leq Z \leq 118$ obtained in the self-consistent calculations. They are compared to experimental data (closed symbols) including the recent data for $Z=114$ and 116. (Taken from S. Ćwiok et al., Phys. Rev. Lett. 83(1999) 1108, and to be published.)

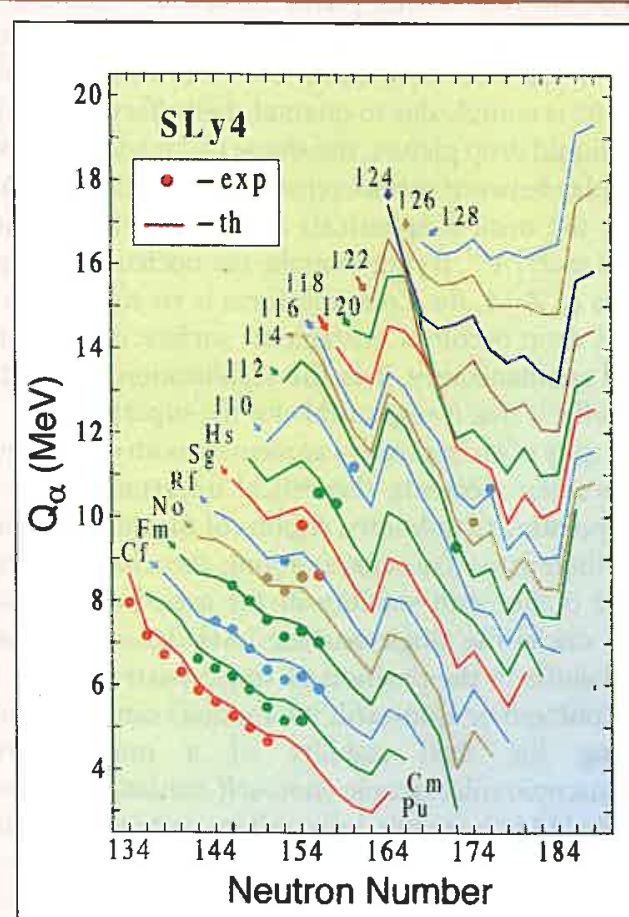




\section{Spinning the heaviest elements}

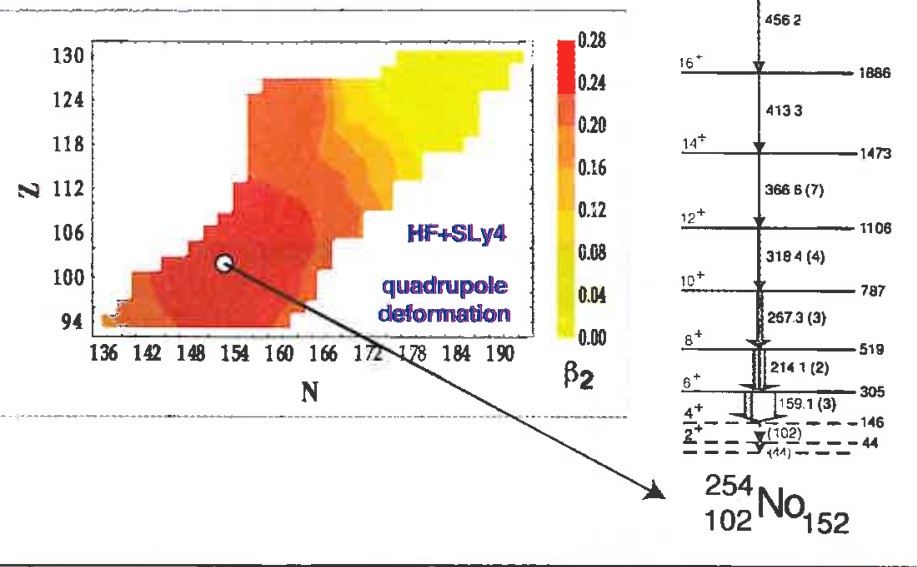

Box 2: In spite of the fact that the number of protons and nucleons in the nucleus is rather small, and nucleonic velocities are fast, atomic nuclei exhibit collective modes such as rotations and vibrations. Many nuclei emit photons characteristic of the sequential de-excitation of rotational bands. In quantum mechanics, collective rotation can only arise for systems that have a deformed shape, i.e., nonspherical distribution of the nucleonic density. It has been only recently that rotational photons could be detected in spectroscopic studies of the transactinides. First results on the collective properties of nobelium isotopes ${ }^{252,254} \mathrm{No}$ have been obtained at Argonne (USA) and Jyväskylä (Finland). It has been found that the ground state rotional band of ${ }^{254} \mathrm{No}$, the best deformed nucleus above ${ }^{208} \mathrm{~Pb}$, resists fission up to a spin of at least $20 \hbar$ (see Fig. 6). Detailed microscopic calculations have shown that the fission barrier of this nucleus changes very weakly with angular momentum, thus explaining this amazing stability of the nobeliums as a combined effect of the Coulomb and Coriolis-plus-centrifugal forces.

Fig. 6: Experimental confirmation of the role of deformation in transactinides was recently obtained for ${ }^{254}$ No. The figure (right portion) shows a cascade of transitions characteristic of the rotation of a deformed nucleus. From the precise energy difference between successive states, it is inferred that ${ }^{254}$ No has a football-like shape with an axis ratio of 4:3, in agreement with theory. The fact that states with angular momenta as high as $20 \hbar$ were detected underscores the remarkable resilience of the shell effects against the centrifugal force and fission, Modern nuclear structure calculations - such as the one labeled HF+Sly4 (left) - tell that stability comes in specific cases from the ability of the nucleus to deform. Here, the deformation parameter $\beta_{2}$ characterizes the elongation of the nuclear shape (for $\beta_{2}=0$ nucleus is spherical).

The calculations predict the competition between several of these forms for the central nuclear density due to the huge electric charge. It is difficult to say at present whether these exotic topologies can occur as metastable states. Will the spherical bubble minimum undergo the shape transition to the "band-like" configuration? What is the stability of all these shapes to reflection-asymmetric and triaxial deformations? It is possible that there exist isolated islands of nuclear stability, associated with very exotic topologies of nuclear density, stabilized by shell effects.

\section{Perspectives}

The heavy element research has been traditionally concentrated in three laboratories: Berkeley, Dubna, and GSI. But the exciting new discoveries brought new players to the "superheavy" community: RIKEN (Japan) and GANIL (France). Chemical studies of transactinides are also being carried out at PSI and JAERI (Japan).

One of the most important and urgent tasks facing experimentalists will be to confirm the new elements found in Dubna. This will not be easy, since these nuclei form an isolated island, disconnected from the known region of the nuclear chart. In order to identify the elements beyond $Z=112$, new tools, such as direct mass measurements or chemical studies must be developed. How to reach even heavier nuclei? The new-generation high-current stable-beam accelerators will certainly be helpful in making new discoveries at a picobarn level. However, in order to explore new, more neutron-rich superheavy regions, accelerated beams of radioactive neutron-rich nuclei will have to be utilized. It is hoped that the new high-powered ISOL facilities will enable us to explore the theoretically crucial region of nuclei between the deformed $N=162$ shell and the spherical $N=184$ shell.

There are also many challenges facing nuclear theory of superheavies. The currently highly phenomenological models used to describe heavy-ion fusion are not reliable when extrapolating outside the regions of known nuclei. The situation is not better for the description of static fission barriers and of dynamics of fission. Slightly better is the situation in the theoretical modeling of nuclear structure of transactinides, where the microscopic self-consistent models can make quantitative predictions. Here, the main problem is how to extrapolate density-dependent effective interactions to the superheavy "terra incognita" and how to describe exotic forms of nuclear existence created thanks to the huge Coulomb force and the borders of the superheavy region. The advances in computer technology and in numerical algorithms will certainly be crucial for the success of this task.

This research was supported in part by the U.S. Department of Energy under Contract Nos. DE-FG02-96ER40963 (University of Tennessee), DE-AC05-00OR22725 with UT-Battelle, LLC (Oak Ridge National Laboratory), the Polish Committee for Scientific Research (KBN) under Contract No. 2 P03B 040 14, and NATO grant PST.CLG.977613.

\section{References}

[1] S. Hofmann and G. Münzenberg, Rev. Mod. Phys. 72 (2000) 733.

[2] S. Ćwiok et al., Nucl. Phys. A611 (1996) 211.

[3] M. Bender, W. Nazarewicz, and P.G. Reinhard, Phys. Lett. B 515 (2001) 42 .

[4] J.V. Kratz, in Heavy Elements and Related New Phenomena, Eds. W. Greiner and R.K. Gupta (World Scientific, Singapore, 1999) p. 129.

[5] P. Schwerdtfeger and M. Seth, Relativistic Effects of the Superheavy Elements, Encyclopedia of Computational Chemistry, Vol. 4 (Wiley, New York, 1998), p. 2480; V.Pershina et al. in Heavy Elements and Related New Phenomena, Eds. W. Greiner and R.K. Gupta (World Scientific, Singapore, 1999) p. 194. 